\title{
Spinocerebellar Ataxia Type 7 Cerebellar Disease Requires the Coordinated Action of Mutant Ataxin-7 in Neurons and Glia, and Displays Non-Cell-Autonomous Bergmann Glia Degeneration
}

\author{
Stephanie A. Furrer, ${ }^{1}$ Mathini S. Mohanachandran, ${ }^{1}$ Sarah M. Waldherr, ${ }^{1}$ Christopher Chang, ${ }^{1}$ Vincent A. Damian, ${ }^{2}$ \\ Bryce L. Sopher, ${ }^{1}$ Gwenn A. Garden, ${ }^{1,4}$ and Albert R. La Spada ${ }^{3,5,6}$ \\ Departments of ${ }^{1}$ Neurology, ${ }^{2}$ Biochemistry, and ${ }^{3}$ Medicine (Medical Genetics), and ${ }^{4}$ The Center on Human Development and Disability, University of \\ Washington, Seattle, Washington 98195, and 5Departments of Pediatrics, Cellular and Molecular Medicine, and Neurosciences, Division of Biological \\ Sciences, and the Institute for Genomic Medicine, University of California, San Diego, La Jolla, California 92093, and 'Rady Children's Hospital, San Diego, \\ California 92123
}

Spinocerebellar ataxia type 7 (SCA7) is a dominantly inherited disorder characterized by cerebellum and brainstem neurodegeneration. SCA7 is caused by a CAG/polyglutamine (polyQ) repeat expansion in the ataxin-7 gene. We previously reported that directed expression of polyQ-ataxin-7 in Bergmann glia (BG) in transgenic mice leads to ataxia and non-cell-autonomous Purkinje cell (PC) degeneration. To further define the cellular basis of SCA7, we derived a conditional inactivation mouse model by inserting a loxP-flanked ataxin-7 cDNA with 92 repeats into the translational start site of the murine prion protein (PrP) gene in a bacterial artificial chromosome (BAC). The PrP-floxed-SCA7-92Q BAC mice developed neurological disease, and exhibited cerebellar degeneration and BG process loss. To inactivate polyQ-ataxin-7 expression in specific cerebellar cell types, we crossed PrP-floxed-SCA7-92Q BAC mice with Gfa2-Cre transgenic mice (to direct Cre to BG) or Pcp2-Cre transgenic mice (which yields Cre in PCs and inferior olive). Excision of ataxin-7 from BG partially rescued the behavioral phenotype, but did not prevent BG process loss or molecular layer thinning, while excision of ataxin-7 from PCs and inferior olive provided significantly greater rescue and prevented both pathological changes, revealing a non-cell-autonomous basis for $\mathrm{BG}$ pathology. When we prevented expression of mutant ataxin-7 in BG, PCs, and inferior olive by deriving Gfa2-Cre;Pcp2-Cre;PrP-floxed-SCA7-92Q $B A C$ triple transgenic mice, we noted a dramatic improvement in SCA7 disease phenotypes. These findings indicate that SCA7 disease pathogenesis involves a convergence of alterations in a variety of different cell types to fully recapitulate the cerebellar degeneration.

\section{Introduction}

Spinocerebellar ataxia type 7 (SCA7) is a progressive, dominantly inherited neurodegenerative disorder characterized by neuron dysfunction, culminating in cell loss in the retina, cerebellum, and brainstem. SCA7 patients consequently exhibit ataxia, motor system abnormalities, blindness, and early mortality. SCA7 is caused by a CAG/polyglutamine (polyQ) repeat expansion in the

Received Aug. 3, 2011; revised Sept. 1, 2011; accepted Sept. 13, 2011.

Author contributions: S.F., B.S., G.G., and A.R.L.S. designed research; S.F., M.M., S.W., C.C., V.D., and B.S. performed research; S.F., V.D., B.S., G.G., and A.R.L.S. analyzed data; S.F., G.G., and A.R.L.S. wrote the paper.

This work was supported by the NIH Grants R01 EY14061 (to A.R.L.), R01 NS52535 (to G.A.G.), GM07108 (to S.A.F.), and UL1-DE19583 (University of California, Davis, Neurotherapeutics Research Institute; to A.R.L.), with facilities support from P30-HD02274 (to the University of Washington Center on Human Development and Disability). We thank R. T. Libby, J. Huang, D. E. Possin, S. K. Custer, J. Eng, R. Holman, and T. Ashe for technical support; and N. Stella and T. D. Baughan for comments on the manuscript.

The authors declare no competing financial interests.

Correspondence should be addressed to either of the following: Dr. Albert R. La Spada, Pediatrics, Neurosciences, and Cellular \& Molecular Medicine, University of California, San Diego, 9500 Gilman Drive, MC 0642, La Jolla, CA 92093-0642, E-mail: alaspada@ucsd.edu; or Dr. Gwenn A. Garden, Department of Neurology, University of Washington, 1959 NE Pacific Street, Box 356465, Seattle, WA 98195, E-mail: gagarden@uw.edu.

DOI:10.1523/JNEUROSCI.4000-11.2011

Copyright $\odot 2011$ the authors $\quad 0270-6474 / 11 / 3116269-10 \$ 15.00 / 0$ coding region of the ataxin-7 gene. As in other diseases caused by polyQ repeat expansion, ataxin-7 is ubiquitously expressed. Nonetheless, inheriting polyQ-expanded ataxin-7 yields degeneration in specific neuronal populations that are selectively vulnerable to this mutation (Garden and La Spada, 2008).

A number of mouse models have been generated to investigate SCA7 disease pathogenesis. PolyQ-expanded ataxin-7, expressed in cerebellar Purkinje cells (PCs) using the Pcp2 promoter, produced very mild ataxia with no impact on life span (Yvert et al., 2000). Using the murine prion protein (PrP) transgenic expression system, we expressed polyQ-expanded ataxin-7 throughout the CNS, but not in PCs. The resulting PrP-SCA7-c92Q mice developed ataxia and $\mathrm{PC}$ degeneration, documenting a role for noncell-autonomous PC degeneration in SCA7 disease pathogenesis (Garden et al., 2002). Transgenic mice that express ataxin-7-92Q protein only in Bergmann glia (BG) in the cerebellum also develop ataxia and PC degeneration, implicating glial dysfunction in polyQataxin-7-mediated PC degeneration (Custer et al., 2006).

While these studies underscored the importance of specific non-neuronal cell types in cerebellar degeneration, PCs are integrated into a complex neural network and receive glutamatergic 
input from axons projecting from the inferior olive (IO) and cerebellar granule cells. BG remove excess glutamate from PC synapses (Huang and Bordey, 2004) and secrete neurotrophic factors, which support PCs (Mount et al., 1995). IO neurons produce insulin-like growth factor 1 , a neurotrophic factor that promotes PC survival and glutamate-aspartate transporter (GLAST) expression in BG (NietoBona et al., 1993; Gamboa and Ortega, 2002).

To clarify the cellular basis of SCA7, we developed a powerful new mouse model wherein polyQ-ataxin-7 expression could be spatially and temporally regulated. In this model, an ataxin-7 92Q cDNA is flanked by loxP sites at the start site of translation in the murine $\mathrm{PrP}$ gene in a bacterial artificial chromosome (BAC). The PrP-floxed-SCA7-92Q BAC mice ubiquitously express mutant ataxin-7 protein in all neurons, including PCs, and develop cerebellar ataxia and histopathology. To determine which cell types are required for SCA7 disease pathogenesis, $\operatorname{PrP-floxed-SCA7-92Q~BAC~}$ mice were crossed with driver lines expressing Cre-recombinase in BG (Gfa2-Cre) or in PCs and IO (Pcp2-Cre). Gfa2-Cre;PrP-floxedSCA7-92Q BAC and Pcp2-Cre;PrP-floxed-SCA7-92Q BAC bigenic mice each displayed a less severe phenotype than PrP-floxed-SCA792Q BAC littermates. Excision of ataxin-7-92Q from PCs and IO neurons not only protected against cerebellar atrophy, but also prevented BG pathology. Excision of ataxin-7-92Q from BG, however, did not prevent these pathological features, suggesting that BG pathology is non-cell autonomous in SCA7. When we created triple transgenic (i.e., Gfa2-Cre; Pcp2-Cre; PrP-floxed-SCA7-92Q BAC) mice, onset of cerebellar ataxia was delayed up to 20 weeks. Together, these findings indicate that BG, PCs, and IO neurons interact in an intimate and tightly interconnected network, and, thus, together contribute to SCA7.

\section{Materials and Methods}

Generation of PrP-floxed-SCA7-92Q BAC mice

We obtained a $90 \mathrm{~kb}$ fragment containing the murine PrP in a BAC vector backbone (RPCI mouse BAC library 24; clone ID RP24-92I12). The murine PrP BAC targeting vector was performed in five steps, as follows: (1) 5' and 3' targeting arms were inserted by PCR onto the ends of a minimal $1.8 \mathrm{~kb}$, chloramphenicol-resistant vector; (2) an ampicillin resistance cassette was cloned into the MluI, XmaI sites of the targeting vector; (3) a 234 bp fragment encoding the last 50 aa of ataxin-7, followed by a loxP site, was inserted into the XhoI, HpaI restriction sites of the targeting vector; (4) an 824 bp fragment encoding a $3 \times$ FLAG tag fused to EGFP was cloned into the NheI, PacI restriction sites of the targeting vector; and (5) a $2.8 \mathrm{~kb}$ fragment containing a loxP site, followed by the remaining ataxin-7-92Q sequence, was cloned into the MluI, XhoI restriction sites to complete the targeting vector. The targeting vector was recombined into the BAC vector backbone and replaced all coding information for the murine PrP gene. The final PrPfloxed-SCA7-92Q BAC vector was microinjected into C57BL/6J:C3H/HeJ F1 hybrid oocytes. Of four founders identified, two gave rise to $\mathrm{PrP}$-floxed$S C A 7-92 Q B A C$ lines with comparable phenotypes. Of these lines, we chose to focus our studies principally on line $258^{\prime}$.

\section{PCR genotyping}

Mice tissue $\left(\sim 2-4 \mathrm{~mm}^{2}\right)$ was placed in a $1.5 \mathrm{ml}$ Microfuge tube containing $175 \mu \mathrm{l}$ of $0.1 \mathrm{M} \mathrm{NaOH}$ and $175 \mu \mathrm{l}$ of mineral oil. Tubes were briefly centrifuged and then heated in an Oster Food Steamer (Target) for 45 $\mathrm{min}$. The tubes were removed from the steamer, inverted several times, and then centrifuged at $13,000 \times g$ in a benchtop centrifuge for $3 \mathrm{~min}$. Eighty microliters of base solution was carefully taken from the middle of the aqueous phase. This material was transferred to a new $0.65 \mathrm{ml} \mathrm{Mi-}$ crofuge tube containing $80 \mu \mathrm{l}$ of $1 \mathrm{M}$ Tris- $\mathrm{HCl}$. This neutralized tissue extract was stored at $4^{\circ} \mathrm{C}$ before PCR analysis. PCR conditions included $60^{\circ} \mathrm{C}$ annealing temperature, 38 cycles, and 1 min extension period. Primer sequences are shown in Table 1.
Table 1. Primer sequences

\begin{tabular}{|c|c|c|}
\hline Transgene & Primer sequence & Product (bp) \\
\hline \multicolumn{3}{|c|}{ PrP-floxed-SCA7-92Q BAC } \\
\hline Forward & CATTTTAGGCCCCACGTATCAC & \multirow[t]{2}{*}{587} \\
\hline Reverse & GGCCCGCTCCGACAT & \\
\hline \multicolumn{3}{|l|}{ CMV-Cre } \\
\hline Forward & САAАATTTGCCTGCATTACCG & \multirow[t]{2}{*}{553} \\
\hline Reverse & САTTCTCCCACCGTCAGTACG & \\
\hline \multicolumn{3}{|l|}{ Z/EG } \\
\hline Forward & GACGACGGCAACTACAAGACC & \multirow[t]{2}{*}{561} \\
\hline Reverse & GCCAGAAGTCAGATGCTCAAG & \\
\hline \multicolumn{3}{|l|}{ Gfa2-Cre } \\
\hline Forward & GTGCTGGGATTACAAGCATGA & \multirow[t]{2}{*}{400} \\
\hline Reverse & CAGGCAAATTTTGGTGTACGG & \\
\hline \multicolumn{3}{|l|}{ Pcp2-Cre } \\
\hline Forward & GATGGATTTCCGTCTCTGGTG & \multirow[t]{2}{*}{428} \\
\hline Reverse & СATTATAAGCTGCAATAAACAAGTTCTGC & \\
\hline
\end{tabular}

\section{RT-PCR analysis}

Mouse brains were homogenized in Trizol (Life Technologies), and total RNA was extracted according to the manufacturer's instructions. Total RNA was reverse transcribed in independent duplicate reactions using random hexamers and SuperScript II reverse transcriptase (Life Technologies), and quantification of mouse and human ataxin-7 RNA expression by coamplification and subsequent restriction digestion was performed as previously described (La Spada et al., 2001). Total RNA was isolated from the cerebellum of 6-week-old mice using an RNeasy isolation kit (Qiagen). Total RNA was reverse transcribed using SuperScriptII Reverse Transcriptase according to manufacturer's instructions (Invitrogen). The cDNAs were subjected to real-time PCR using custom ataxin-7 primers and probe selected with Primer Express v3.0 software (Applied Biosystems), as follows: forward primer, TTTGGGAGCCGGCAGAT; reverse primer, GCTGTGCATTCAGATGCTTCTC; probe, TGTTTGACTCCAGGTGGA. Samples were analyzed on a StepOnePlus RealTime PCR system using a $64^{\circ} \mathrm{C}$ annealing temperature and normalized to mouse 18S RNA using Applied Biosystems assay Hs99999901_s1. Average ataxin-7 RNA level for nontransgenic and PrP-floxed-SCA7-92Q $B A C$ mice ( $n \geq 3$ /group) was calculated using Microsoft Excel. Ataxin-7 levels in nontransgenic animals was normalized to 1.0, and data were plotted and statistically analyzed in Graphpad Prism 4.0.

\section{Behavioral phenotyping}

All studies and procedures were approved by the University of Washington and University of California, San Diego, Institutional Animal Care and Use Committees (IACUC). Females and males were used in all studies, and experiments were performed by a blinded examiner.

Composite phenotype score. The composite phenotype score was performed as previously described (Guyenet et al., 2010). An average composite phenotype score for each genotype was calculated and plotted using Microsoft Excel. Statistical analyses were conducted in Graphpad Prism 4.0.

Accelerating rotarod. Mice were placed on the rotarod (Economex, Columbia Instruments) and trained on day 0 by running freely up to 5 min. On days $1-4$, mice were placed on the rotarod and underwent the following regimen: at time 0 , the rotarod was started with four rotations per minute, and an acceleration of 0.1 rotations per second. The time each mouse dismounted was recorded (in seconds), and mice had at least 5 min of rest between trials. Trials were repeated five times daily for a maximum of $300 \mathrm{~s}$ per trial. The best four times per day were recorded and averaged for each mouse. Average latency to fall for each trial day was calculated for each genotype and plotted using Microsoft Excel. Statistical analyses were conducted in Graphpad Prism 4.0.

\section{Immunohistochemistry}

Deeply anesthetized mice were transcardially perfused with PBS followed by $4.0 \%$ paraformaldehyde (PFA) in $0.1 \mathrm{M}$ phosphate buffer (PB), $\mathrm{pH}$ 7.4. Brains were removed and postfixed in $4.0 \%$ PFA in $0.1 \mathrm{M} \mathrm{PB}$ overnight, then moved into $0.4 \%$ PFA in $0.1 \mathrm{M} \mathrm{PB}$ for storage at $4^{\circ} \mathrm{C}$. Free-floating 40 $\mu \mathrm{m}$ sagittal brain sections were cut on the Leica Vibratome $1000 \mathrm{~S}$ and 
A
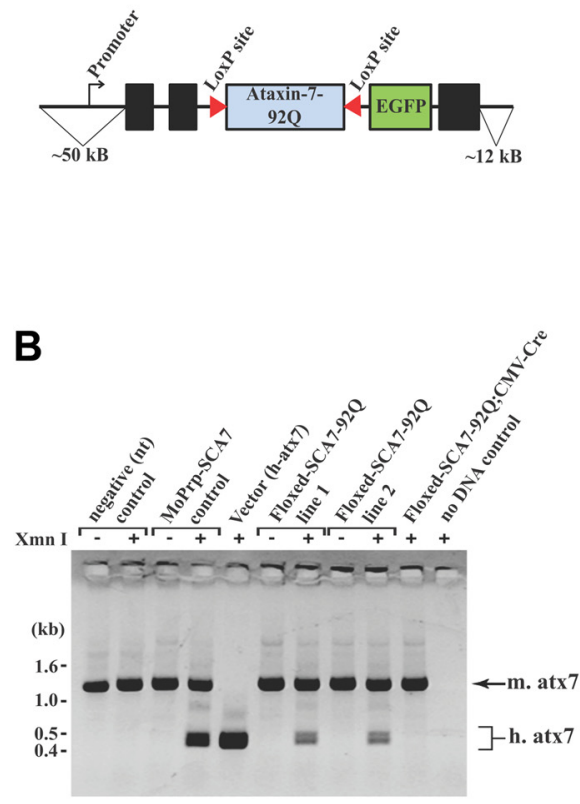

C

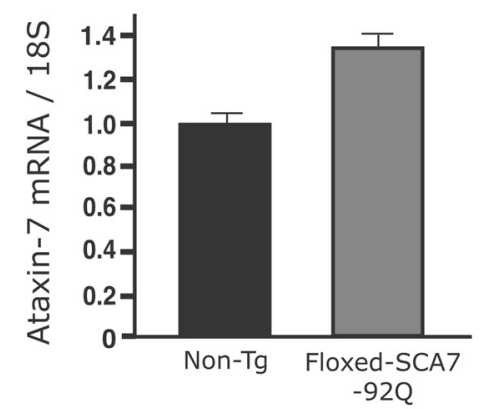

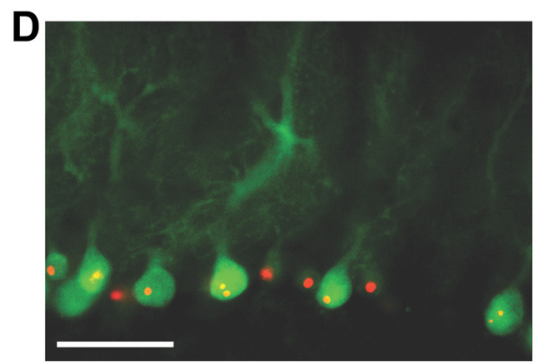

$\mathbf{E}$
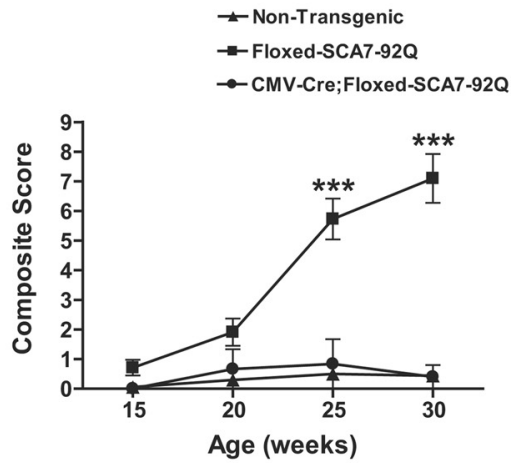

$\mathbf{F}$
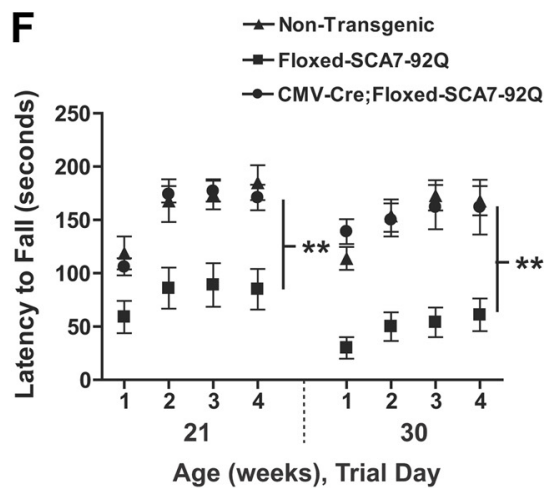

Figure 1. PrP-floxed-SCA7-92Q BAC mice exhibit Cre-dependent ataxin-7 expression and disease phenotype. $A$, Diagram of the PrPfloxed-SCA792Q BAC construct, indicating the location of the human ataxin-7920 CDNA flanked by loxP sites, a downstream EGFP reporter gene, and the first two exons and last exon from the murine prion protein gene (black boxes; not to scale). $\boldsymbol{B}$, We performed RT-PCRanalysis of cerebella with primers that coamplified murine and human ataxin-7, and then differentiated mouse endogenous ataxin-7 ( $\mathrm{m}$. atx7) from human transgenic ataxin-7 (h. atx7) by Xmnl restriction digestion. PrP-floxed-SCA7-92Q transgene-positive mice yielded Xmnl-cut bands, which were no longer present after crossing with CMV-Cre mice that ubiquitously express (re recombinase. C, We performed quantitative RT-PCR analysis of cerebellar RNA with primers that coamplified murine and human ataxin-7, normalized to mouse 185 RNA levels, for nontransgenic and PrP-floxed-SCA7-92Q BAC mice ( $n=3 /$ group). We set the total ataxin-7 level to 1 in the nontransgenic control and found that the total ataxin-7 level in the PrP-floxed-SCA7-920 BAC mice is 1.3, indicating that the BAC transgene is expressed at lower than endogenous levels. $\boldsymbol{D}$, Immunofluorescent labeling of ataxin-7 (red) and calbindin (green) in cerebellar sections from 40-week-old PrP-floxed-SCA7-92Q BAC mice. Scale bar, $50 \mu \mathrm{m}$. E, We completed composite phenotype analysis for sets of nontransgenic $(n=14)$, PrP-floxed-SCA7-920 BAC $(n=10)$, and CMV-Cre:PrP-floxed-SCA7-920 BAC $(n=5)$ mice. PrP-floxed-SCA7-920 BAC mice develop a significant phenotype, which is eliminated upon Cre-mediated excision ( ${ }^{* * *} p<0.001$, two-way ANOVA with Bonferroni's post hoc test). Error bars indicate SEM. $\boldsymbol{F}$, We measured the mean latency to fall from the accelerating rotarod for sets of nontransgenic $(n=10)$, PrP-floxed-SCA7-92Q BAC $(n=10)$, and CMV-Cre;PrP-floxed-SCA7-92Q BAC $(n=5)$ mice. PrP-floxed-SCA7-92Q BAC mice performed significantly worse on the rotarod at 21 and 30 weeks of age compared with nontransgenics, and CMV-Cre;PrP-floxed-SCA7-92Q BAC mice performed much better than PrP-floxed-SCA7-92Q BAC mice at these time points $\left({ }^{* *} p<0.01\right.$, two-way ANOVA with Bonferroni's post hoc test). Error bars indicate SEM.

stored in $0.03 \%$ PFA in PBS. Free-floating sections were incubated with $5 \%$ normal goat serum (NGS), $0.2 \%$ BSA, and $1.0 \%$ Triton X-100 for $1 \mathrm{~h}$ at room temperature. Sections were then incubated overnight at $4^{\circ} \mathrm{C}$ with anti-calbindin antibody (300, Swant) at 1:1000 dilution; anti-GFP antibody (ab6556, Abcam) at 1:1000 dilution; anti-GFAP antibody (Z0334, DAKO) at 1:1000 dilution; anti-S100 $\beta$ antibody (ab11178, Abcam) at 1:1000 dilution; or anti-ataxin-7 antibody (PA1-749, Affinity Bioreagents) at 1:500 dilution in PBS with 5\% NGS and 0.1\% Triton X-100. After three washes in $1 \times$ PBS, AlexaFluor 488 anti-rabbit or anti-mouse IgG (Invitrogen) at 1:500 dilution, and/or AlexaFluor 594 anti-rabbit or anti-mouse IgG (Invitrogen) at 1:500 dilution were applied to sections and incubated at room temperature for 2-3 h. After three washes in $1 \times$ PBS, sections were mounted on glass slides and coverslipped in Fluoro-Gel with Tris Buffer (Electron Microscopy Sciences). Sections were viewed and 2-D or 3-D $z$-stack digital images were created using a Zeiss Axiovert 200M inverted microscope and Slidebook 5.0 software (Intelligent Imaging Innovations).

To quantify PrP-floxed-SCA7-92Q BAC gene excision, 2-D images were collected from three areas per section, one section per animal, for Gfa2-Cre;Z/EG and Z/EG animals $(n=$ 6/group). 2-D images were collected of four areas per section, one section per animal, for Pcp2-Cre;PrP-floxed-SCA7-92Q BAC $(n=7)$ and PrP-floxed-SCA7-92Q BAC $(n=4)$ mice. Two independent observers counted the number of either calbindin-positive Purkinje cells or S- $100 \beta$-positive Bergmann glia, then counted the number of those that were GFP positive. Results were reported as a ratio of GFP and marker double-positive cells to total marker-positive cells. Average ratios for each genotype were calculated, plotted, and analyzed statistically in Graphpad Prism 4.0.

To quantify cerebellar molecular layer thickness, 2-D images of calbindin-immunostained cerebellar sections were collected. The three most dorsal cerebellar folia per section, using three sections per individual ( $n=6$ /group), were imaged. In each image, a boundary line around the molecular layer from, but excluding, the Purkinje cell bodies to the pial surface was drawn by hand using Slidebook 5.0. In the center of the molecular layer, $100-\mu \mathrm{m}$-long lines were drawn. Every $100 \mu \mathrm{m}$, a measurement was taken from the pial surface to the Purkinje cell layer. Molecular layer measurements were averaged for each genotype, plotted, and analyzed statistically in Graphpad Prism 4.0.

To quantify the number of Bergmann glia processes, brightest-point projection images were created using Slidebook 5.0. 3-D image $z$-stacks were collected for two areas per section, three sections per individual ( $n=6$ /group). 3-D images were deconvolved using the Nearest Neighbors algorithm of Slidebook 5.0. The brightest-point projection images were created through the $z$-axis to produce 2-D images. In each 2-D image, a $150 \mu \mathrm{m}$ line was drawn using Slidebook 5.0 (Intelligent Imaging Innovations) $\sim 50 \mu \mathrm{m}$ from the pial surface of the cerebellar molecular layer. Three independent observers drew this line and then counted the number of GFAP-positive processes that crossed the line. The average number of processes for each genotype was calculated, plotted, and analyzed statistically in Graphpad Prism 4.0.

\section{Results}

Characterization of the PrP-floxed-SCA7-92Q BAC transgenic mouse

To generate a mouse model that can delineate cell type-specific contributions to SCA7 disease pathogenesis, we inserted an ataxin-7 cDNA, carrying 92 CAG repeats and flanked by loxP sites, into a murine prion protein $\mathrm{BAC}$, and placed an EGFP reporter gene 
downstream of the ataxin-7 cDNA (Fig. 1A). After transgenic microinjection of this BAC construct, we identified founders and established two independent PrP-floxedSCA7-92Q BAC lines. RT-PCR analysis of cerebellar RNA from $P r P$-floxed-SCA7-92Q $B A C$ mice demonstrated that both $\operatorname{PrP}$ floxed-SCA7-92Q BAC lines expressed human ataxin-7 (Fig. 1B). Quantitative real-time RT-PCR analysis of ataxin-7 on RNA extracted from cerebellum confirmed that transgene expression was less than endogenous ataxin-7 expression (Fig. 1C). To ensure Cre recombinase-mediated excision of the ataxin-7 cDNA, we crossed $\mathrm{PrP}$ floxed-SCA7-92Q BAC mice with CMV-Cre transgenic mice. The resulting $C M V-C r e$; PrP-floxed-SCA7-92Q BAC bigenic mice no longer expressed human ataxin-7, confirming successful Cre-mediated bigenic mice no longer expressed human excision (Fig. 1B). Immunofluorescent labeling of $\mathrm{PrP}$ floxed-SCA7-92Q BAC brain sections with an anti-ataxin-7 antibody revealed widespread expression of the mutant protein, as evidenced by aggregate formation in neurons, including granule cell neurons (GCNs) and Purkinje cell neurons (Fig. 1D). PrP-floxed-SCA7-92Q BAC mice did not exhibit a significant reduction in calbindin-labeled PC soma (2.6 $\pm 0.1 \mathrm{PCs}$ per $100 \mu \mathrm{m}$; \pm SEM) compared with nontransgenic mice $(3.0 \pm 0.2$ PCs per 100 $\mu \mathrm{m} ; \pm \mathrm{SEM})(p=0.13$, unpaired twotailed $t$ test), similar to previously published mouse models of SCA7 (Garden et al., 2002; Chou et al., 2010).

Motor behavior in PrP-floxed-SCA7-92Q BAC mice was assessed using a composite phenotype scoring system (Guyenet et al., 2010) and by rotarod analysis. Beginning at 20 weeks of age, PrP-floxedSCA7-92Q BAC mice exhibited a more severe behavioral phenotype than nontransgenic mice, based upon composite phenotype score, and this progressively worsened with age (Fig. 1E). PrP-floxedSCA7-92Q BAC mice also performed significantly worse on the accelerating rotarod at 21 weeks of age compared with nontransgenic littermates, and their rotarod performance worsened at 30 weeks of age (Fig. $1 F$ ). CMV-Cre-driven excision of the ataxin-7 cDNA prevented these phenotypes in CMV-Cre;PrP-floxed-SCA7-92Q BAC bigenic mice, as these bigenic mice performed comparably to nontransgenic mice (Fig. $1 E, F$ ). Together, these results indicate that the PrP-floxed-SCA7-92Q BAC transgenic mice develop a SCA7 phenotype characterized by progressive ataxia and impaired motor function. Furthermore, the utility of this model for studying cell-type contribution was confirmed by widespread Cre-mediated excision of the mutant ataxin-7 gene.

\section{PolyQ-ataxin-7 expression in Bergmann glia is required for the full SCA7 phenotype}

We have previously shown that expression of polyQ-expanded ataxin-7 in BG, driven by a human glial fibrillary acidic protein promoter known as Gfa2' (Besnard et al., 1991), is sufficient to produce SCA7 cerebellar neurodegeneration (Custer et al., 2006). To determine whether mutant ataxin-7 expression in BG is re-
C

- Non-Transgenic

$\rightarrow-$ Floxed-SCA7-920

$\rightarrow$ Gfa2-Cre

$\rightarrow$ Gfa2-Cre; Floxed-SCA7-92Q
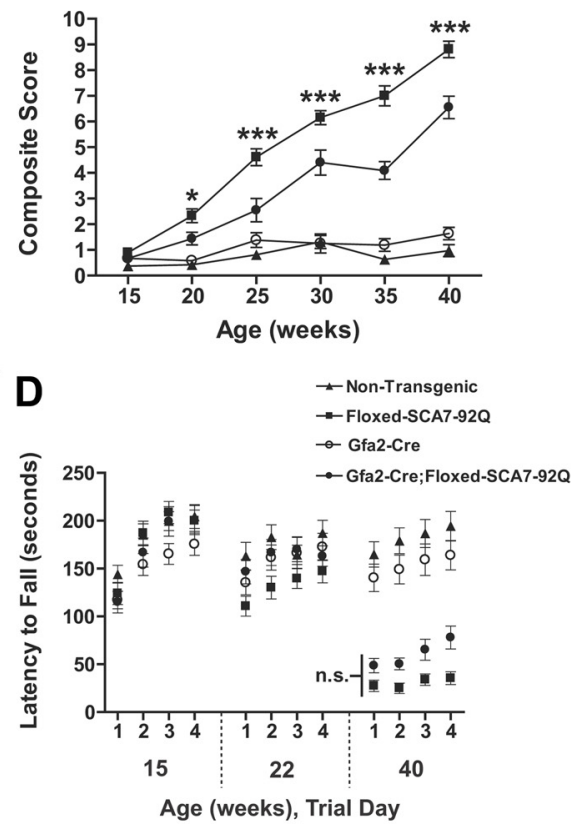

Age (weeks), Trial Day

Figure 2. Excision of polyQ-ataxin-7 from Bergmann glia partially ameliorates SCA7 neurological phenotypes. $\boldsymbol{A}$, Immunofluorescence labeling of EGFP (green) and S100- $\beta$ (red) in Gfa2-Cre;Z/EG cerebellar sections. B, Gfa2-Cre expression produced a 72\%

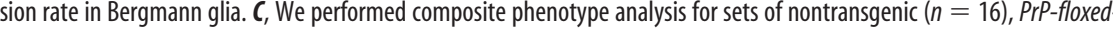
-920 BAC mice perform significantly worse than nontransgenic and Gfa2-Cre mice at 40 weeks of age ( $p<0.001$, tw0-way ANOVA with Bonferroni's post hoc test). Gfa2-Cre;PrP-floxed-SCA7-92Q BAC mice exhibited a trend toward improved rotarod performance, but this was not significant. Error bars indicate SEM.

quired for the full SCA7 phenotype, we crossed a mouse line expressing Cre recombinase driven by the Gfa2 promoter (Zhuo et al., 2001) with the PrP-floxed-SCA7-92Q BAC mice. In $\operatorname{PrP}$ floxed-SCA7-92Q BAC mice, EGFP expression is induced upon Cre-mediated excision of the loxP flanked ataxin-7 cDNA, as EGFP is moved adjacent to the murine prion protein promoter. In Gfa2-Cre; PrP-floxed-SCA7-92Q BAC mice, EGFP expression is low and BG cell bodies are very compact, complicating determination of the excision rate in bigenic BG. Thus, to validate this driver line, we crossed Gfa2-Cre mice with a robust Z/EG reporter line (Novak et al., 2000). Sagittal brain sections from Gfa2-Cre; $Z / E G$ mice were immunolabeled for GFP and $S 100-\beta$, a BG cell body marker. We found that $72 \%$ of all S100- $\beta$-positive BG were concurrently positive for GFP (Fig. $2 A, B$ ), validating the utility of the Gfa2-Cre driver line for high-level BG excision.

We assessed the behavioral phenotype of Gfa2-Cre;PrPfloxed-SCA7-92Q BAC bigenic mice by again using the composite phenotype score and accelerating rotarod. As expected, $\operatorname{Pr} P$ floxed-SCA7-92Q BAC mice displayed significantly higher composite phenotype scores, beginning at 20 weeks of age (Fig. $2 C$ ). Gfa2-Cre;PrP-floxed-SCA7-92Q BAC mice, however, displayed a progressive, but less severe phenotype compared with $P r P$-floxedSCA7-92Q BAC littermates. On the accelerating rotarod, $\operatorname{Pr} P$ floxed-SCA7-92Q BAC mice performed poorly at 22 weeks of age and were significantly impaired by 40 weeks of age (Fig. 2D). Gfa2-Cre;PrP-floxed-SCA7-92Q BAC mice performed poorly on 
A

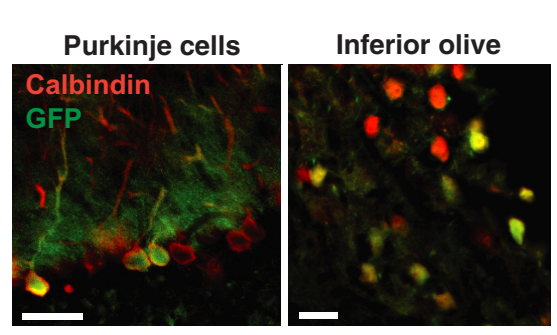

B
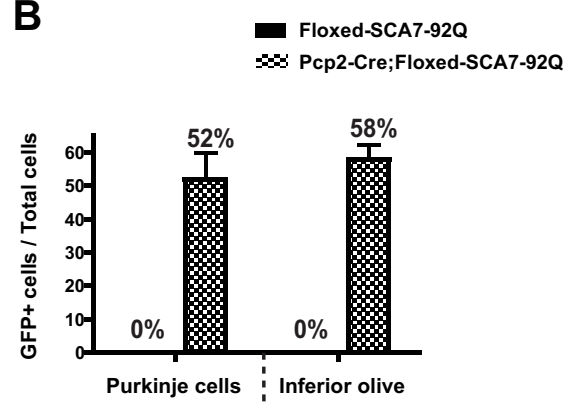

C

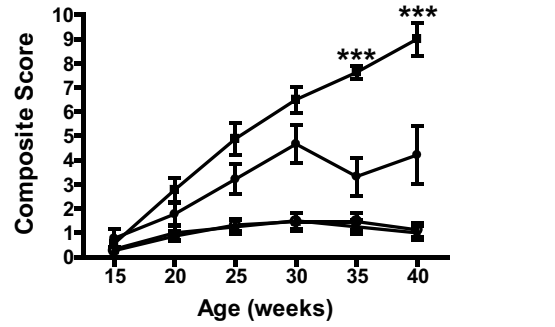

D

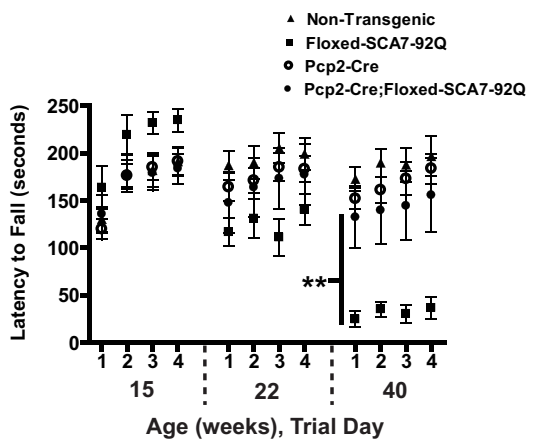

Figure 3. Excision of polyQ-ataxin-7 from Purkinje cells and inferior olive partially ameliorates SCA7 neurological phenotypes. $A$, Immunofluorescence labeling of EGFP (green) and calbindin (red) in Pcp2-Cre;PrP-floxed-SCA7-92Q BAC bigenic cerebellum and brainstem sections. Scale bars, $50 \mu \mathrm{m}$. B, Pcp2-driven Cre expression yielded a 52\% excision rate in Purkinje cells and a $58 \%$ excision rate in inferior olive neurons. $C$, Composite phenotyping revealed that $P C p 2$-Cre; $P r P$-floxed-SCA7-92Q BAC mice $(n=9)$ are moderately improved compared with PrP-floxed-SCA7-92Q BAC mice $(n=8)$ from 20 to 30 weeks of age, but not compared with nontransgenic $(n=12)$ or Pcp2-Cre $(n=15)$ controls. Moreover, the neurological phenotype of Pcp2-Cre;PrP-floxed-SCA7-92Q $B A C$ mice ceases to progress after 30 weeks of age, and thus becomes dramatically improved compared with their progressing PrP-floxed-SCA7-92Q BAC littermates at these time points ( ${ }^{* * *} p<0.001$, two-way ANOVA with Bonferroni's post hoc test). Error bars indicate SEM. D, PCp2-Cre;PrP-floxed-SCA7-92Q BAC mice exhibit a trend toward improved rotarod performance at 22 weeks of age. By 40 weeks of age, however, PCp2-Cre;PrP-floxed-SCA7-92Q BAC mice are markedly superior to their PrP-floxed-SCA7-92Q $B A C$ littermates, and actually perform comparably to nontransgenic and Pcp2-Cre controls on the accelerating rotarod $\left({ }^{* *} p<\right.$ 0.01, two-way ANOVA with Bonferroni's post hoc test). Error bars indicate SEM.

the accelerating rotarod at 40 weeks as well, but tended to remain on the rotarod longer than their PrP-floxed-SCA7-92Q $B A C$ littermates (Fig. 2D). Together, these data suggest that polyQ-expanded ataxin-7 expression in BG contributes to SCA7 pathogenesis and is required to recapitulate the full SCA7 behavioral phenotype.

\section{PolyQ-ataxin-7 expression in inferior olive is required for SCA7 disease phenotype}

Restricted expression of polyQ-expanded ataxin-7 in cerebellar PCs in transgenic mice is not sufficient to cause progressive motor impairment (Yvert et al., 2000). Indeed, ataxia and PC degeneration can develop in SCA7 mice in which mutant ataxin-7 is expressed throughout the brain, but not in PCs (Garden et al., 2002), indicating the importance of non-cell-autonomous degeneration in SCA7. To test whether excising mutant ataxin-7 in PCs would significantly alter the disease phenotype, we crossed PrP-floxed-SCA7-92Q BAC mice with a Purkinje cell protein 2 (Pcp2)-Cre line, obtained from the Jackson Laboratory (Barski et al., 2000). When we assessed the excision rate in Pcp2-Cre;PrPfloxed-SCA7-92Q BAC bigenic mice by immunofluorescent labeling with antibodies to EGFP and calbindin, we noted that 52\% of all calbindin-positive PCs were also EGFP positive (Fig. $3 A, B$ ). Importantly, in the course of performing this analysis, we docu- mented that expression of Cre in this line is not limited to PCs, but is also present in the inferior olive, the superior olive, and the olfactory bulb (Fig. 3A; data not shown). These studies thus reconfirmed the integrity of the PrP-floxed-SCA7-92Q $B A C$ construct, as singly transgenic $\operatorname{Pr} P$ floxed-SCA7-92Q cerebellar sections showed no EGFP immunoreactivity, indicating that EGFP expression is only released upon Cre-mediated gene excision (Fig. 3B).

Neurons of the IO degenerate in SCA7 patients (Garden and La Spada, 2008) as well as in SCA7 transgenic mice (Wang et al., 2010). The IO projects climbing fibers (CFs) that form glutamatergic synapses on PC somae and dendrites. The IO also delivers the neurotrophic factor insulin-like growth factor-1 (IGF-1) to PCs (NietoBona et al., 1993). Thus, loss of climbing fiber input to PCs may contribute to noncell-autonomous PC degeneration in SCA7. When we quantified the percentage of calbindin-positive IO neurons that also express GFP to determine excision rate, as was just described for PCs, we found that ataxin-7 cDNA excision had occurred in $58 \%$ of IO neurons (Fig. 3A,B). Composite phenotype scoring indicated that $P c p 2-C r e$; PrP-floxed-SCA7-92Q BAC bigenic mice had a significantly milder phenotype than their singly transgenic SCA7 littermates, and, interestingly, neurological impairment in Pcp2-Cre;PrP-floxed-SCA7-92Q $B A C$ mice reached a plateau at 30 weeks of age (Fig. 3C). Accelerating rotarod analysis at 22 weeks of age indicated that $\operatorname{PrP}$-floxedSCA7-92Q BAC mice displayed evidence of motor incoordination at this time, while Pcp2-Cre;PrP-floxed-SCA7-92Q BAC bigenic mice performed comparably to nontransgenic and Pcp2-Cre controls (Fig. 3D). By 40 weeks of age, the motor performance differences became quite dramatic, as Pcp2-Cre;PrP-floxed-SCA7-92Q BAC bigenic mice remained on the rotarod at least four times longer than their $\operatorname{Pr} P$ floxed-SCA7-92Q BAC littermates. Together, these findings demonstrate that expression of mutant ataxin-7 in IO neurons, and likely also in PCs, is required to recapitulate the full SCA7 phenotype.

PolyQ-ataxin-7 excision in Purkinje cells and inferior olive, but not in Bergmann glia, protects against cerebellar molecular layer degeneration

A prominent feature of SCA7 neurodegeneration in transgenic mice is shortened PC dendritic arbors in the cerebellar molecular layer (Garden et al., 2002; Yoo et al., 2003; Custer et al., 2006; Chou et al., 2010). To investigate whether this loss is mitigated in Gfa2-Cre;PrP-floxed-SCA7-92Q BAC or Pcp2-Cre;PrP-floxedSCA7-92Q BAC bigenic mice, we immunostained sagittal cerebellar sections with anti-calbindin antibody. By 40 weeks of age, we noted reduced dendritic calbindin immunoreactivity, abnormal PC morphology, and molecular layer thinning in the cerebellum of PrP-floxed-SCA7-92Q BAC mice (Fig. 4A,B). Gfa2-Cre;PrPfloxed-SCA7-92Q BAC bigenic mice also exhibited reduced calbindin immunoreactivity and a thinner molecular layer, similar to 
PrP-floxed-SCA7-92Q BAC mice (Fig. 4C); however, Pcp2-Cre;PrP-floxed-SCA7-92Q $B A C$ cerebellar sections resembled cerebellar sections from nontransgenic controls (Fig. 4A-D).

To further investigate the effect of conditional ataxin-7 excision from selected cell populations in the cerebellum, we quantified molecular layer thickness in dorsal cerebellum. When we measured the width of the molecular layer along the outside of each folium from the pial surface to the PC layer boundary at $100 \mu \mathrm{m}$ intervals (Fig. $4 E$ ), we found that molecular layer thickness was significantly reduced from an average of $92.8 \mu \mathrm{m}$ in nontransgenic controls to $75.5 \mu \mathrm{m}$ in $\mathrm{PrP}$ floxed-SCA7-92Q BAC mice (Fig. 4F). Although Gfa2-Cre;PrP-floxed-SCA7-92Q $B A C$ mice had a similarly reduced molecular layer thickness, Pcp2-Cre;PrP-floxedSCA7-92Q BAC bigenic mice exhibited molecular layer thickness values that were comparable to nontransgenic controls and markedly increased versus PrP-floxedSCA7-92Q BAC mice (Fig. 4F). The molecular layer thickness in Gfa2-Cre and Pcp2Cre singly transgenic mice did not differ from nontransgenic animals (data not shown). These findings indicate that excision of mutant ataxin-7 from PCs and IO neurons rescues $\mathrm{PC}$ neurodegeneration in SCA7 transgenic mice, while excision of mutant ataxin-7 from BG does not prevent such PC pathology.

\section{PolyQ-ataxin-7 excision in Purkinje cells and inferior olive, but not in Bergmann glia, prevents Bergmann glia degeneration}

As BG exhibit morphological pathology and dysfunction in SCA7 (Garden et al., 2002; Custer et al., 2006), we examined BG morphology in PrP-floxed-SCA7-92Q BAC mice and the two bigenic lines. After staining sagittal cerebellar sections for GFAP to visualize BG processes, we generated 3-D images of dorsal cerebellar folia, deconvolved them, and transformed them into 2-D images by creating brightest-point projections. This analysis revealed that PrP-floxed-SCA7-92Q BAC mice have remarkably fewer BG processes in the cerebellar molecular layer (Fig. $5 A, B$ ). Although the morphology of BG in Gfa2-Cre;PrP-floxed-SCA7-92Q BAC bigenic mice resembled that of PrP-floxed-SCA7-92Q BAC mice, BG in Pcp2-Cre;PrP-floxed-SCA7-92Q BAC mice exhibited more prominent processes, indicative of an intermediate pathology between nontransgenic controls and PrP-floxed-SCA7-92Q BAC mice (Fig. $5 A-D$ ). To quantify BG pathology, we counted the number of GFAP-positive BG processes that crossed a $150 \mu \mathrm{m}$ line drawn parallel to, and $50 \mu \mathrm{m}$ from, the pial surface (Fig. $5 E$ ). PrP-floxed-SCA7-92Q BAC mice harbored significantly fewer BG processes than nontransgenic controls (Fig. $5 F$ ). Surprisingly, BG processes were also markedly reduced in Gfa2-Cre;PrP-floxedSCA7-92Q BAC mice, similar to PrP-floxed-SCA7-92Q BAC mice. BG process number in Pcp2-Cre;PrP-floxed-SCA7-92Q $B A C$ bigenic mice was not significantly different from nontransgenic mice or from PrP-floxed-SCA7-92Q BAC mice, indicative
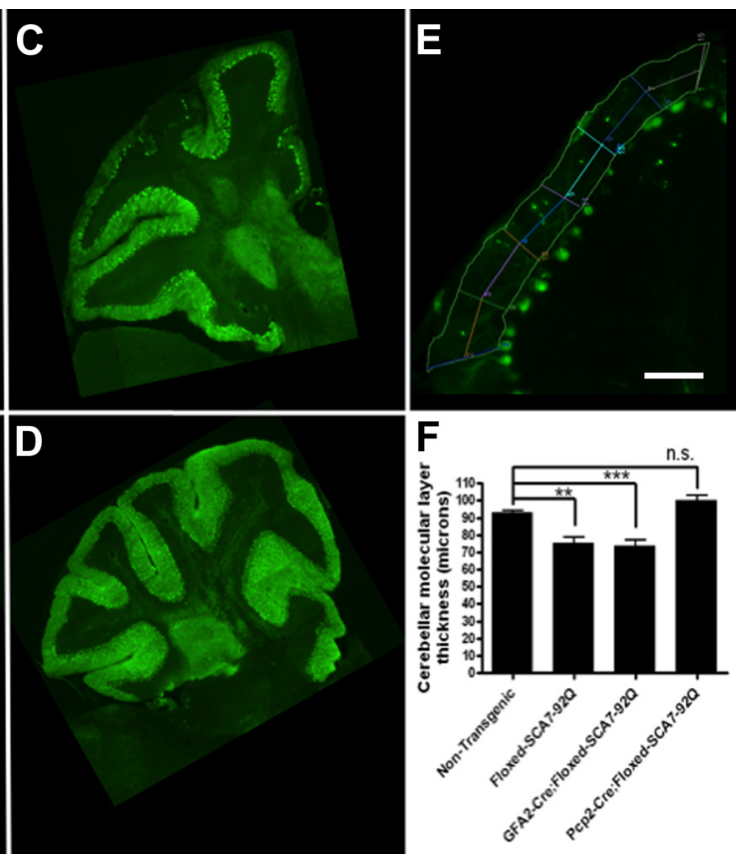

Figure 4. Excision of polyQ-ataxin-7 from Purkinje cells and inferior olive prevents cerebellar degeneration in PrP-floxed-SCA7BC mice. $\boldsymbol{A}-\boldsymbol{D}$, Anti-calbindin antibody staining of cerebellar sections. Calbindin immunoreactivity in nontransgenic contro

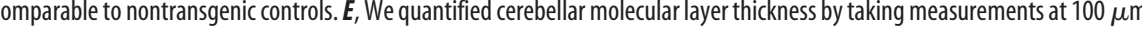

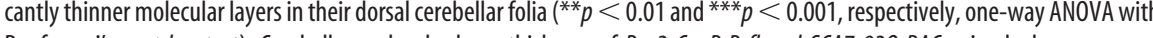
Bonferroni's post hoc test). Cerebellar molecular layer thickness of Pcp2-Cre;PrP-floxed-SCA7-92Q BAC animals, however, was trols. Error bars indicate SEM. Scale bars: $\boldsymbol{A}, 300 \mu \mathrm{m} ; \boldsymbol{E}, 100 \mu \mathrm{m}$

of an intermediate phenotype (Fig. $5 F$ ). Neither Gfa2-Cre nor Pcp2-Cre singly transgenic mice differed from nontransgenic controls (data not shown). Hence, excision of mutant ataxin-7 from PCs and IO neurons ameliorated BG histopathology, while elimination of mutant ataxin-7 expression from BG did not improve BG degeneration. This finding represents the first demonstration that BG dysfunction and degeneration in SCA7 is noncell autonomous.

PolyQ-ataxin-7 excision from Bergmann glia, Purkinje cells, and inferior olive delays SCA7 disease onset

Because excising polyQ-ataxin-7 from BG, or from PCs and IO neurons, partially rescued SCA7 neurodegeneration, we proceeded to test whether eliminating mutant ataxin-7 from all three cell types would further ameliorate SCA7 disease pathogenesis. To do so, we generated triple transgenic mice that carried the PrP-floxed-SCA7-92Q BAC, the Pcp2-Cre transgene, and the Gfa2-Cre transgene. Composite phenotype analysis revealed that such triple transgenic mice exhibit very late disease onset, but, once manifested, neurological abnormalities are progressive (Fig. 6A). Rotarod analysis, however, indicated that triple transgenic mice retain normal motor coordination function and perform dramatically better than singly transgenic PrP-floxed-SCA7-92Q BAC mice at 40 weeks of age (Fig. $6 \mathrm{~B}$ ).

To quantify the delay in onset, we pooled data from all $\operatorname{PrP}$ floxed-SCA7-92Q BAC, nontransgenic, Gfa2-Cre;PrP-floxedSCA7-92Q BAC, Pcp2-Cre;PrP-floxed-SCA7-92Q BAC, and triple transgenic mice analyzed during the course of this study. 

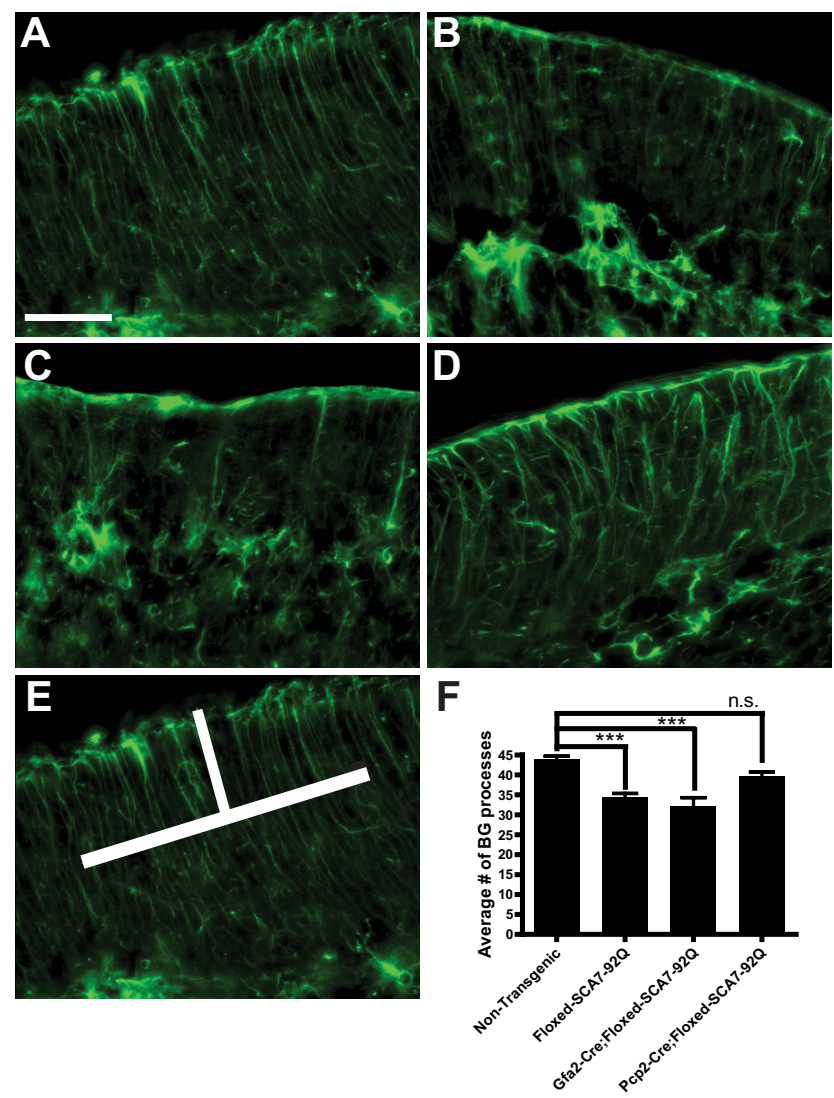

Figure 5. Excision of polyQ-ataxin-7 from Purkinje cells and inferior olive, but not Bergmann glia, prevents loss of glial processes. A-D, Anti-GFAP antibody staining of dorsal cerebellar folia. Comparison of nontransgenic controls $(A)$ to PrP-floxed-SCA7-92Q $B A C$ mice $(\boldsymbol{B})$ demonstrates an obvious reduction in Bergmann glia processes in the PrPfloxed-SCA7-92Q BAC mice; Gfa2-Cre;PrP-floxed-SCA7-92Q BAC cerebellar sections (C) resembled PrP-floxed-SCA7-92Q BAC sections, while Pcp2-Cre;PrP-floxed-SCA7-92Q BAC cerebellar sections $(\boldsymbol{D})$ appeared comparable to nontransgenic controls. $\boldsymbol{E}$, We quantified Bergmann glia processes by counting the number of GFAP-positive processes that cross a $150 \mu \mathrm{m}$ line drawn parallel to, and $\sim 50 \mu \mathrm{m}$ from, the pial surface. $\boldsymbol{F}$, Results of quantifications of Bergmann glia processes ( $n=6 /$ group). PrP-floxed-SCA7-92Q BAC and Gfa2Cre;PrP-floxed-SCA7-92Q BAC animals have significantly fewer Bergmann glia processes compared with nontransgenic mice $\left({ }^{* * *} p<0.001\right.$, one-way ANOVA with Bonferroni's post hoc test). However, the number of Bergmann glia processes in Pcp2-Cre;PrP-floxedSCA7-92Q BAC mice is intermediate between nontransgenic controls and PrP-floxed-SCA7$920 B A C$ mice. Error bars indicate SEM. Scale bar, $30 \mu \mathrm{m}$.

One-way ANOVA testing, followed by a Bonferroni's multiplecomparison post hoc analysis, was conducted for all composite phenotype data. This analysis showed that PrP-floxed-SCA7-92Q $B A C$ mice display neurological abnormalities at 20 weeks of age $(p<0.001)$, establishing the age of onset. Both Gfa2-Cre;PrPfloxed-SCA7-92Q BAC and Pcp2-Cre;PrP-floxed-SCA7-92Q BAC bigenic mice exhibit significant neurological impairments by 25 weeks of age ( $p<0.01$ and $p<0.001$, respectively). The triple transgenic mice, however, do not develop statistically significant neurological deficits until 40 weeks of age $(p<$ 0.01 ), a delay of onset of 20 weeks. Thus, SCA7 mice, in which the ataxin-7 transgene is excised from BG, PCs, and IO neurons, exhibit a dramatically improved phenotype, underscoring the combined importance of all these components of the cerebellar circuitry.

To further assess the effect of removing mutant ataxin-7 expression from BG, PCs, and IO neurons, we analyzed the histological phenotype of the trigenic animals. Mean cerebellar
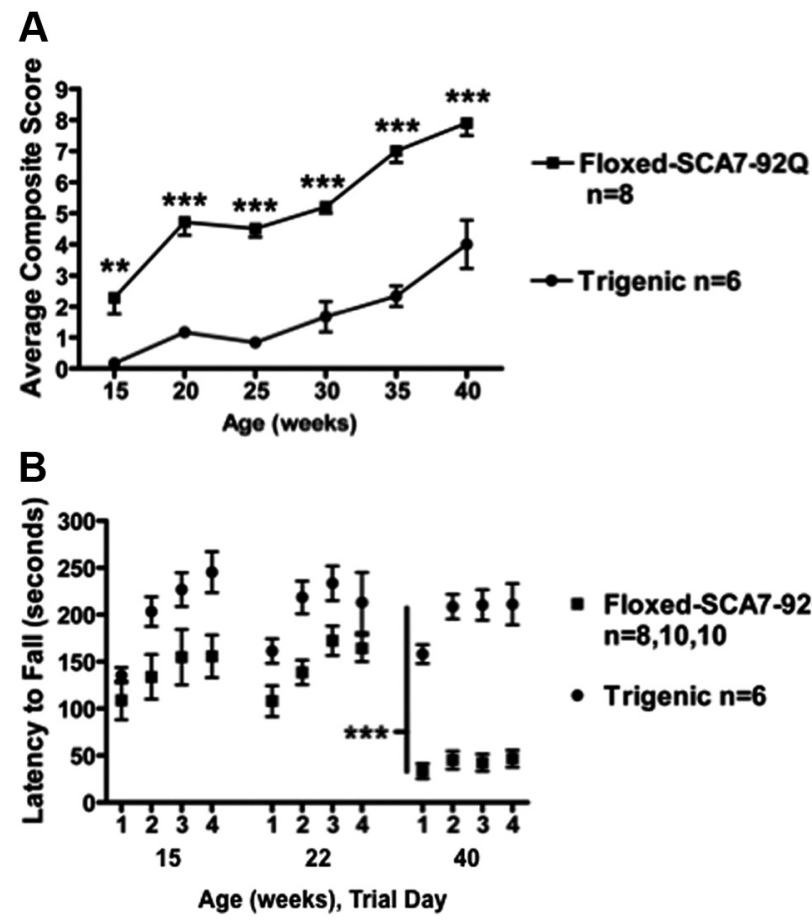

Figure 6. Excision of polyQ-ataxin-7 from Bergmann glia, Purkinje cells, and inferior olive greatly retards neurological disease onset in PrP-floxed-SCA7-92Q BAC mice. A, Composite phenotype scoring of Gfa2-Cre;Pcp2-Cre;PrP-floxed-SCA7-92Q BAC (trigenic) mice reveals markedly delayed onset of a progressive behavioral phenotype, and superior performance scores compared with PrP-floxed-SCA7-92QBAC mice at all time points tested $\left({ }^{* *} p<0.01,{ }^{* * *} p<0.001\right.$ two-way ANOVA with Bonferroni's post hoc test; $n=6-8$ /group). Error bars indicate SEM. $B$, Trigenic mice consistently performed better on the accelerating rotarod than PrP-floxed-SCA7$920 B A C$ mice at all time points; and rotarod performance was dramatically superior at 40 weeks of age ( ${ }^{* * *} p<0.001$, two-way ANOVA with Bonferroni's post hoc test; $n=6-10 /$ group). Error bars indicate SEM.

molecular layer thickness of the triple transgenic mice $(100.2$ $\mu \mathrm{m})$ did not significantly differ from that measured in the Pcp2Cre;PrP-floxed-SCA7-92Q bigenic mice $(100.0 \mu \mathrm{m})$. To address the possibility that mutant ataxin-7-mediated degeneration of cerebellar granule cell neurons could contribute to the SCA7 phenotype, we analyzed the cerebellar granule cell layer in nontransgenic and PrP-floxed-SCA7-92Q BAC mice. No overt morphological changes were noted, and the width of the granule layer was modestly increased in PrP-floxed-SCA7-92Q BAC mice compared with nontransgenic controls (data not shown). These results indicate that delay of disease onset until 40 weeks of age in Gfa2-Cre;Pcp2-Cre; PrP-floxed-SCA7-92Q BAC transgenic mice is best attributed to incomplete excision of ataxin-7 cDNA from PCs, IO neurons, and BG.

\section{Discussion}

In this study, we report a new SCA7 mouse model in which ataxin-7 gene expression can be controlled by Cre recombinase. In this model, a murine PrP BAC containing $>50 \mathrm{~kb}$ of DNA $5^{\prime}$ to the $\operatorname{PrP}$ translational start site produced widespread expression of ataxin-7-92Q, including in PC neurons. In a prior study, a smaller PrP promoter fragment lacking key regulatory elements to drive broad expression of ataxin-7-92Q was used, but expression in PCs was not observed (Garden et al., 2002). PrP-floxedSCA7-92Q BAC mice developed progressive ataxia and cerebellar degeneration typical of other SCA7 models (Garden et al., 2002; Yoo et al., 2003; Custer et al., 2006; Wang et al., 2010). When PrP-floxed-SCA7-92Q BAC mice were crossed with mice with 
ubiquitous Cre expression, mutant ataxin-7 was no longer produced and no behavioral phenotype developed, validating the loxP sites. Excision of ataxin-7-92Q from BG in the cerebella of Gfa2-Cre;PrP-floxed-SCA7-92Q BAC bigenic mice significantly ameliorated neurological abnormalities measured by composite phenotype scoring (Guyenet et al., 2010), but did not prevent cerebellar molecular layer thinning or BG process loss. Excision of ataxin-7-92Q from PCs and IO neurons improved motor behavior and protected against cerebellar molecular layer thinning and BG process loss. These findings indicate that expression of mutant ataxin-7 in BG is not required for glial degeneration. However, expression of mutant ataxin-7 in PCs and IO neurons contributes to BG pathology. Thus, SCA7 exhibits non-cell-autonomous BG degeneration, revealing for the first time that neuronal health directly impacts BG in SCA7.

Studies over the last decade support the realization that the cellular basis of neurodegenerative disorders is not simply cell autonomous. An important example comes from a series of studies on familial amyotrophic lateral sclerosis (ALS) due to dominant, gain-of-function mutations in superoxide dismutase 1 (SOD1). This form of ALS has been extensively studied since its genetic basis was defined in 1993 (Rosen et al., 1993). Transgenic expression of disease-causing SOD1 mutations in mice recapitulated key features of human ALS motor neuron degeneration (Gurney et al., 1994). These early mouse models used the native SOD1 promoter, resulting in ubiquitous expression of the mutant SOD1 protein. When transgenic strategies relying upon neuron-restricted expression of mutant SOD1 failed to produce significant motor neuron disease in mice (Pramatarova et al., 2001; Lino et al., 2002), many groups theorized that motor neuron degeneration might be non-cell autonomous in this form of ALS. Production of chimeric mice containing different mixtures of normal and mutant-expressing SOD1 cells allowed investigators to definitively show that motor neuron expression of mutant SOD1 is not sufficient for disease (Clement et al., 2003). Instead, non-neuronal cells must express mutant SOD1 for neighboring motor neurons to degenerate. Current models of ALS postulate that a variety of different molecular pathologies contribute to the motor neuron degeneration by eliciting damage within different cell types, and that death of the motor neuron does not occur in isolation from its connected glial, vascular, and immune system partners (Ilieva et al., 2009).

In SCA7, it is also clear that neurodegeneration is not simply cell autonomous. Our study examined the roles of BG, PCs, and IO neurons, and found that all of these cell types contribute to SCA7 PC degeneration. We previously reported that expression of mutant ataxin-7 in BG in Gfa2-SCA7-92Q transgenic mice is sufficient to cause PC degeneration (Custer et al., 2006). In the current study, removal of ataxin-7-92Q from PCs and IO neurons prevented PC degeneration (as measured by cerebellar molecular layer thinning), despite continued expression of mutant ataxin-7 protein in BG. One likely explanation for the difference between these two studies is that PrP-floxed-SCA7-92Q BAC mice express mutant ataxin-7 at levels less than that of the endogenous gene, while expression in Gfa2-SCA7-92Q transgenic mice exceeded endogenous levels. Furthermore, isolated expression of mutant ataxin-7 in BG resulted in late-onset ataxia and PC degeneration in the Gfa2-SCA7-92Q model. When mutant ataxin-7 is expressed ubiquitously at lower levels, reduction of mutant ataxin-7 expression in PCs and IO neurons is more beneficial than excision of mutant ataxin-7 in BG. Thus, expression of mutant ataxin-7 in IO neurons, in combination with expression in

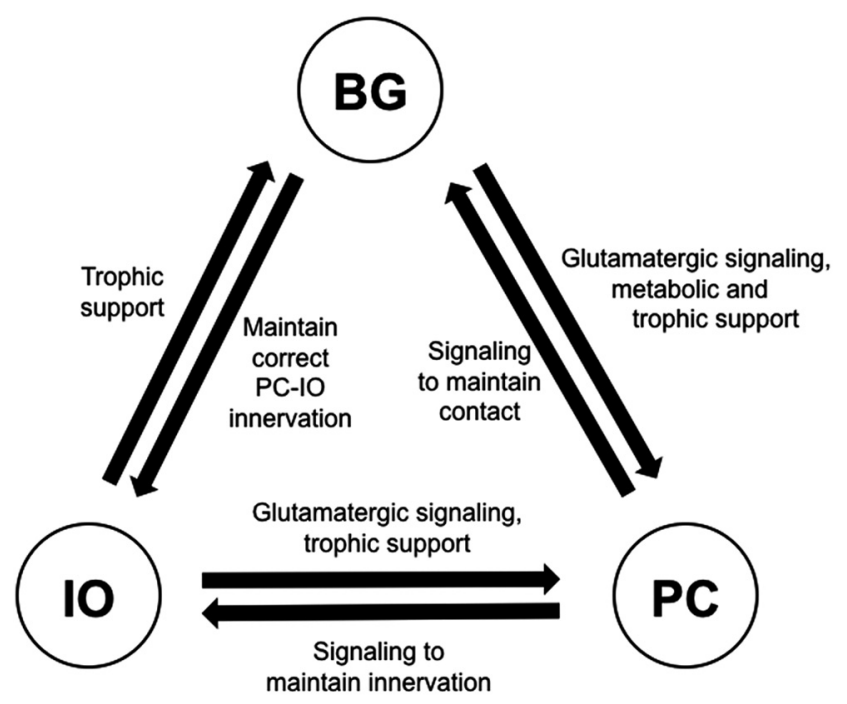

Figure 7. Interdependent connections between $B G, P C$, and 10 account for the multi-cell type origin of SCA7 neurodegeneration. BG, PCs, and I0 neurons each engage in bilateral interactions to yield an interdependent neural-glial network in which the function and survival of all cell types are assured together. During SCA7 disease pathogenesis, dysfunction of one cell type will inevitably impact the other connected cell types due to loss of trophic support and/or release of excitotoxic or inflammatory mediators. Altered function in one cell type thus culminates in all cell types failing to maintain appropriate signaling or other key functions, thereby exacerbating damage in the initial cell type while propagating abnormalities in the other connected cell types.

PCs, produces greater cerebellar toxicity than mutant ataxin-7 expression in BG. Nonetheless, our current findings continue to support an important role for BG in SCA7 pathogenesis. Excision of the disease gene from $\mathrm{BG}$ alone does ameliorate the disease phenotype, and excision of mutant ataxin-7 from BG in combination with PCs and IO neurons yields a synergistic effect on the delay of symptom onset.

An important and unexpected observation in this study is that a well established Pcp2-Cre transgenic driver line (Barski et al., 2000) expresses Cre-recombinase in both PCs and IO neurons. This permitted us to evaluate the contribution of IO neurons and PCs to cerebellar degeneration in SCA7, but prevented us from delineating the individual role of each cell type in SCA7 pathogenesis. Previous studies in transgenic mice have shown that polyQ-ataxin-7 expression restricted to PCs produces a very mild, late-onset phenotype (Yvert et al., 2000). However, expression in other cerebellar cell types, but not in PCs, yields more severe SCA7 disease (Garden et al., 2002; Custer et al., 2006). Together, these studies, in conjunction with data presented here, suggest that IO neuron dysfunction is a major contributor to SCA7 disease pathogenesis. A principal role for IO neurodegeneration in SCA7 is suggested not only by our findings, but also by work done independently on a different SCA7 transgenic mouse model (Wang et al., 2010).

How might IO neuron dysfunction result in cerebellar degeneration in SCA7? One function of IO neurons is to deliver IGF-1, an important survival factor for PCs, to the cerebellar molecular layer (Nieto-Bona et al., 1993). Exogenous IGF-1 ameliorates PC degeneration (Tolbert and Clark, 2003; Carrascosa et al., 2004) and promotes glial glutamate transporter gene expression in BG (Gamboa and Ortega, 2002). Impaired GLAST transcription has been documented in SCA7 transgenic mice (Custer et al., 2006), thus loss of IGF-1 delivery from IO neuron CFs may contribute to reduced GLAST gene expression in SCA7. Loss of CFs may also 
explain the observation that the GluR $\delta 2$ AMPA receptor subunit is upregulated in cerebellar lysates from ataxin-7-52Q transgenic mice (Chou et al., 2010). GluR $\delta 2$ is found exclusively at PCparallel fiber (PC-PF) synapses (Kurihara et al., 1997). Deletion of GluR $\delta 2$ in mice causes reduced PC-PF synapses and abnormal extra PC-CF synapses (Kashiwabuchi et al., 1995). It is possible that loss of CF input due to cell-autonomous degeneration of IO neurons leads to increased PC-PF synapses and thus increased GluR $\delta 2$ expression (Chou et al., 2010). Another explanation might involve transcriptional dysregulation resulting in increased GluR $\delta 2$ expression, increased PC-PF synapses, and subsequent reduction of PC-CF synapses, contributing to degeneration of IO neurons. BG are a component of both the $\mathrm{PC}-\mathrm{PF}$ and $\mathrm{PC}-\mathrm{CF}$ tripartite synapse; thus, disruption of inputs to PCs may in turn cause BG dysfunction and/or degeneration. Because mutant ataxin-7 disrupts normal gene transcription (McMahon et al., 2005; Palhan et al., 2005; Helmlinger et al., 2006), it is also possible that the molecular pathways involved in maintaining appropriate connections between PCs, IO neurons, and BG are altered by transcriptional dysregulation in a cell type-specific fashion. It will be critical to identify the cellular processes that mutant ataxin-7 disturbs within each cell type, and how these abnormalities lead to disruptions in the PC-IO neuron-BG network (Fig. 7).

Triple transgenic mice harboring the PrP-floxed-SCA7-92Q $B A C$, Gfa2-Cre, and Pcp2-Cre transgenes exhibited a dramatic rescue from neurological disease and PC degeneration. Remarkably, removing mutant ataxin-7 from these cell populations delayed disease onset by 20 weeks (i.e., twofold). Although disease pathology in the triple transgenic mice could stem from expression of mutant ataxin-7 in cerebellar GCNs, we carefully evaluated GCN morphology, but did not observe any GCN abnormalities, suggesting instead that incomplete excision of mutant ataxin-7 expression from PCs, IO neurons, and BG likely accounts for the ultimate development of a disease phenotype in these mice. Our results demonstrate that PCs, IO neurons, and BG are all involved in SCA7 disease pathogenesis (Fig. 7). PolyQ-ataxin-7 consequently leads to dysfunction in each of these different cell types, preventing them from performing key functions or maintaining healthy connections with their interdependent neighbors. Cerebellar neurodegeneration in SCA7 may thus be viewed as a coincident set of pathological alterations that occur within a highly interconnected network composed of neurons and glia. Our findings underscore the complexity of SCA7 disease pathogenesis and suggest that reversing toxicity in a single cell type is unlikely to reverse the course of disease. However, these findings suggest that effective gene-silencing strategies delivered to any involved cell type should yield significant benefit to afflicted patients.

\section{References}

Barski JJ, Dethleffsen K, Meyer M (2000) Cre recombinase expression in cerebellar Purkinje cells. Genesis 28:93-98.

Besnard F, Brenner M, Nakatani Y, Chao R, Purohit HJ, Freese E (1991) Multiple interacting sites regulate astrocyte-specific transcription of the human gene for glial fibrillary acidic protein. J Biol Chem 266:18877-18883.

Carrascosa C, Torres-Aleman I, Lopez-Lopez C, Carro E, Espejo L, Torrado S, Torrado JJ (2004) Microspheres containing insulin-like growth factor I for treatment of chronic neurodegeneration. Biomaterials 25:707-714.

Chou AH, Chen CY, Chen SY, Chen WJ, Chen YL, Weng YS, Wang HL (2010) Polyglutamine-expanded ataxin-7 causes cerebellar dysfunction by inducing transcriptional dysregulation. Neurochem Int 56:329-339.

Clement AM, Nguyen MD, Roberts EA, Garcia ML, Boillée S, Rule M, McMahon AP, Doucette W, Siwek D, Ferrante RJ, Brown RH Jr, Julien JP,
Goldstein LS, Cleveland DW (2003) Wild-type nonneuronal cells extend survival of SOD1 mutant motor neurons in ALS mice. Science 302:113-117.

Custer SK, Garden GA, Gill N, Rueb U, Libby RT, Schultz C, Guyenet SJ, Deller T, Westrum LE, Sopher BL, La Spada AR (2006) Bergmann glia expression of polyglutamine-expanded ataxin-7 produces neurodegeneration by impairing glutamate transport. Nat Neurosci 9:1302-1311.

Gamboa C, Ortega A (2002) Insulin-like growth factor-1 increases activity and surface levels of the GLAST subtype of glutamate transporter. Neurochem Int 40:397-403.

Garden GA, La Spada AR (2008) Molecular pathogenesis and cellular pathology of spinocerebellar ataxia type 7 neurodegeneration. Cerebellum $7: 138-149$

Garden GA, Libby RT, Fu YH, Kinoshita Y, Huang J, Possin DE, Smith AC, Martinez RA, Fine GC, Grote SK, Ware CB, Einum DD, Morrison RS, Ptacek LJ, Sopher BL, La Spada AR (2002) Polyglutamine-expanded ataxin-7 promotes non-cell-autonomous Purkinje cell degeneration and displays proteolytic cleavage in ataxic transgenic mice. J Neurosci 22:4897-4905.

Gurney ME, Pu H, Chiu AY, Dal Canto MC, Polchow CY, Alexander DD, Caliendo J, Hentati A, Kwon YW, Deng HX (1994) Motor neuron degeneration in mice that express a human $\mathrm{Cu}, \mathrm{Zn}$ superoxide dismutase mutation. Science 264:1772-1775.

Guyenet SJ, Furrer SA, Damian VM, Baughan TD, La Spada AR, Garden GA (2010) A simple composite phenotype scoring system for evaluating mouse models of cerebellar ataxia. J Vis Exp pii:1787.

Helmlinger D, Hardy S, Abou-Sleymane G, Eberlin A, Bowman AB, Gansmüller A, Picaud S, Zoghbi HY, Trottier Y, Tora L, Devys D (2006) Glutamine-expanded ataxin-7 alters TFTC/STAGA recruitment and chromatin structure leading to photoreceptor dysfunction. PLoS Biol 4:e67.

Huang H, Bordey A (2004) Glial glutamate transporters limit spillover activation of presynaptic NMDA receptors and influence synaptic inhibition of Purkinje neurons. J Neurosci 24:5659-5669.

Ilieva H, Polymenidou M, Cleveland DW (2009) Non-cell autonomous toxicity in neurodegenerative disorders: ALS and beyond. J Cell Biol 187:761-772.

Kashiwabuchi N, Ikeda K, Araki K, Hirano T, Shibuki K, Takayama C, Inoue Y, Kutsuwada T, Yagi T, Kang Y (1995) Impairment of motor coordination, Purkinje cell synapse formation, and cerebellar long-term depression in GluR $\delta 2$ mutant mice. Cell 81:245-252.

Kurihara H, Hashimoto K, Kano M, Takayama C, Sakimura K, Mishina M, Inoue Y, Watanabe M (1997) Impaired parallel fiber-Purkinje cell synapse stabilization during cerebellar development of mutant mice lacking the glutamate receptor $\delta 2$ subunit. J Neurosci 17:9613-9623.

La Spada AR, Fu YH, Sopher BL, Libby RT, Wang X, Li LY, Einum DD, Huang J, Possin DE, Smith AC, Martinez RA, Koszdin KL, Treuting PM, Ware CB, Hurley JB, Ptácek LJ, Chen S (2001) Polyglutamine-expanded ataxin-7 antagonizes CRX function and induces cone-rod dystrophy in a mouse model of SCA7. Neuron 31:913-927.

Lino MM, Schneider C, Caroni P (2002) Accumulation of SOD1 mutants in postnatal motoneurons does not cause motoneuron pathology or motoneuron disease. J Neurosci 22:4825-4832.

McMahon SJ, Pray-Grant MG, Schieltz D, Yates JR $3^{\text {rd }}$, Grant PA (2005) Polyglutamine-expanded spinocerebellar ataxia-7 protein disrupts normal SAGA and SLIK histone acetyltransferase activity. Proc Natl Acad Sci U S A 102:8478-8482.

Mount HT, Dean DO, Alberch J, Dreyfus CF, Black IB (1995) Glial cell line-derived neurotrophic factor promotes the survival and morphologic differentiation of Purkinje cells. Proc Natl Acad Sci U S A 92:9092-9096.

Nieto-Bona MP, Garcia-Segura LM, Torres-Aleman I (1993) Orthograde transport and release of insulin-like growth factor I from the inferior olive to the cerebellum. J Neurosci Res 36:520-527.

Novak A, Guo C, Yang W, Nagy A, Lobe CG (2000) Z/EG, a double reporter mouse line that expresses enhanced green fluorescent protein upon Cremediated excision. Genesis 28:147-155.

Palhan VB, Chen S, Peng GH, Tjernberg A, Gamper AM, Fan Y, Chait BT, La Spada AR, Roeder RG (2005) Polyglutamine-expanded ataxin-7 inhibits STAGA histone acetyltransferase activity to produce retinal degeneration. Proc Natl Acad Sci U S A 102:8472-8477.

Pramatarova A, Laganière J, Roussel J, Brisebois K, Rouleau GA (2001) Neuron-specific expression of mutant superoxide dismutase 1 in 
transgenic mice does not lead to motor impairment. J Neurosci 21:3369-3374.

Rosen DR, Siddique T, Patterson D, Figlewicz DA, Sapp P, Hentati A, Donaldson D, Goto J, O'Regan JP, Deng HX (1993) Mutations in Cu/Zn superoxide dismutase gene are associated with familial amyotrophic lateral sclerosis. Nature 362:59-62.

Tolbert DL, Clark BR (2003) GDNF and IGF-1 trophic factors delay hereditary Purkinje cell degeneration and the progression of gait ataxia. Exp Neurol 183:205-219.

Wang HL, Chou AH, Lin AC, Chen SY, Weng YH, Yeh TH (2010) Polyglutamine-expanded ataxin-7 upregulates Bax expression by activating p53 in cerebellar and inferior olivary neurons. Exp Neurol 224:486-494.
Yoo SY, Pennesi ME, Weeber EJ, Xu B, Atkinson R, Chen S, Armstrong DL, Wu SM, Sweatt JD, Zoghbi HY (2003) SCA7 knockin mice model human SCA7 and reveal gradual accumulation of mutant ataxin-7 in neurons and abnormalities in short-term plasticity. Neuron 37:383-401.

Yvert G, Lindenberg KS, Picaud S, Landwehrmeyer GB, Sahel JA, Mandel JL (2000) Expanded polyglutamines induce neurodegeneration and transneuronal alterations in cerebellum and retina of SCA7 transgenic mice. Hum Mol Genet 9:2491-2506.

Zhuo L, Theis M, Alvarez-Maya I, Brenner M, Willecke K, Messing A (2001) hGFAP-cre transgenic mice for manipulation of glial and neuronal function in vivo. Genesis 31:85-94. 